\title{
ACQUISITION AND MODELING OF A SPECTRUM IN A NON THERMAL EQUILIBRIUM PLASMA FORMED IN AIR WITH WATER
}

\author{
P.André*, Yu.Barinov**, G Faure*, V.Kaplan**, S.Shkol'nik**
}

* LAEPT, Blaise Pascal University, UMR 6069 CNRS, bât. Physique 5, 24 avenue des Landais 63177 Aubière Cedex, France

** A.F. Ioffe Physical-Technical Institute, Russian Academy of Sciences, Polytechnicheskaya 26, St Petersburg, 194021, Russia

\begin{abstract}
Spectra in the range of $365-385 \mathrm{~nm}$, corresponding to the Schumann-Runge system of $\mathrm{O}_{2}$ $\left(\mathrm{B}^{3} \Sigma_{\mathrm{u}}^{-}-\mathrm{X}^{3} \Sigma_{\mathrm{g}}^{-}\right)$and to the second positive system of $\mathrm{N}_{2}\left(\mathrm{C}^{3} \Pi_{\mathrm{u}}-\mathrm{B}^{3} \Pi_{\mathrm{g}}\right)$ are measured by optical emission spectroscopy in a discharge with liquid non-metallic electrodes (DLNME) in air with water at atmospheric pressure. They are simulated theoretically. This discharge generates a non-equilibrium plasma. In this paper, we first describe the experimental set up. Then, we explain the method of spectra simulation. By comparison of measured and simulated spectra, we determine the rotational and vibrational temperature and the ratio between densities of two upper electronic quantum levels of the two considered systems. The possible mechanism of spectra formation is discussed.
\end{abstract}

\section{Key words}

Schumann-Runge system of $\mathrm{O}_{2}$, second positive system of $\mathrm{N}_{2}$, non equilibrium plasma, discharge with liquid non-metallic electrodes, air, water, plasma.

\section{Introduction}

Interest for radiation studies has been revived in the field of plasma physics by the upcoming entry of probes into planet atmosphere (Titan, Mars...) [1, 2] and by industrial applications (circuit breakers, waste treatment...) [3, 4]. The number of papers concerning the plasma modeling has increased the last years $[5,6]$. Unfortunately it appears a lack of data and many areas need further studies. For example to expand the radiation database especially those in ultraviolet and deep ultraviolet wavelength, to study the effect of radiation on 
ionization equilibrium, to test the composition calculation code in non equilibrium plasma, etc. For this purpose, we bring some new spectroscopy data obtained in non equilibrium plasma formed in air and water vapor at atmospheric pressure. Moreover, we prolong our investigation of a new kind of discharge: the discharge with liquid non-metallic electrodes (DLNME). As a matter of fact DLMNE with tap water electrodes is an effective generator of non-equilibrium plasma at the pressures up to atmospheric pressure. The DLMNE burns steadily under a DC power supply and the construction of the discharge assembly is simple: special chamber is not required and the discharge burn in open air. DLMNE generates plasma contaminated by a water vapor with an amount of 20 to $45 \%$ (molar percentage) $[7,8]$.

In this paper, we first describe the discharge assembly, the main features of the discharge and the scheme of experimental set-up for the spectroscopic measurements at the ultraviolet and deep ultraviolet wavelengths. Secondly, we describe the diatomic spectra calculation of the second positive system of $\mathrm{N}_{2}$ and the Schumann-Runge system of $\mathrm{O}_{2}$ in the wavelength range of 365 to $385 \mathrm{~nm}$. The method of calculation was used in [9] to simulate the second positive system of $\mathrm{N}_{2}$. Here it is evolved and applied to simulate the Schumann-Runge system of $\mathrm{O}_{2}$ too. Thirdly, we apply it to the simulation of the experimental results to obtain the rotational and vibrational temperatures. We determine the ratio between densities of two upper electronic quantum levels of the two considered systems $\mathrm{O}_{2}$ and $\mathrm{N}_{2}$. Finally, we discuss the results.

\section{Experimental set-up and discharge parameters.}

The experiments were carried out with the discharge between two tap water streams in open air. The water streams flow along ceramic chutes making a small angle with vertical. The metallic current leads were inserted into the streams and were covered by a water layer with a thickness of a few millimeters. The inter-electrode gap $L$ (the air gap between water surfaces facing each other) can be changed from $L=6 \mathrm{~mm}$ up to $L=10 \mathrm{~mm}$. The discharge axis $z$ is directed from the anode to the cathode and put $z=0$ at the surface of the water anode (Fig.1). The discharge burnt steadily with a DC current supply in a volumetric (diffuse) form at a relatively high voltage and low current density. For example, at current $I=(50-100) \mathrm{mA}$ (current density $\left.j \sim(0.25-0.5) \mathrm{A} / \mathrm{cm}^{2}\right)$ and discharge length $L=6 \mathrm{~mm}$ the voltage between 
metallic leads was $V \sim 3 \mathrm{kV}$. Voltage drop across the water electrodes was of about half of this value. So, the voltage drop across the air gap was $\sim 1.5 \mathrm{kV}$.

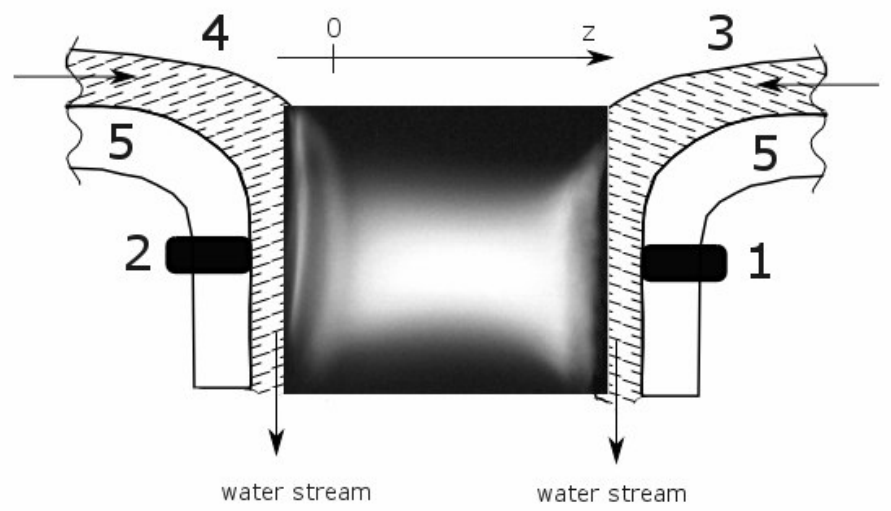

Figure.1: section view of the discharge assembly. 1,2 metallic current leads; 3 watercathode; 4 water-anode; 5 ceramics chutes.

In the previous experiments $[7,8]$, we investigated by means of electric probe, microwave radiation absorption and emission spectroscopy the discharge with a length $L \approx 6 \mathrm{~mm}$ in the same assembly. Obtained results were compared with the spectra simulation and were used for plasma composition calculations.

Our investigations show that the discharge can be distinctly separated into three regions: the near-cathode $(\sim 2.5 \mathrm{~mm}$ in length) and near-anode $(\sim 1.5 \mathrm{~mm}$ in length) regions and the column. The electric field in the column is approximately constant $E_{c} \approx(7-8) \times 10^{2} \mathrm{~V} / \mathrm{cm}$ but the column is not quite uniform. It somewhat narrows at the joint with the near cathode region and expands toward the anode. The gas in the column is heated up to the temperature $\approx 2000 \mathrm{~K}$ in the cathode end and $\approx 1500 \mathrm{~K}$ in the anode end and is contaminated by the water vapor. Water contamination was estimated as $\sim(30-40) \%$ and $\sim(15-25) \%$ in the cathode and in the anode ends of the column correspondingly. So, the parameter $E / N_{g}$ changes from $\approx 20 \mathrm{Td}$ to $\approx 15 \mathrm{Td}$ (here $N_{g}-$ is a heavy particles concentration). The mean value of the charged particle concentration $n_{c}$ is about $10^{12} \mathrm{~cm}^{-3}$. The concentration decreases at the anode end of the column 1.5-2 times as much as against the cathode one. The mean energy of electrons was estimated to be about $0.4 \mathrm{eV}$. The near electrode regions are significantly nonuniform. The mean value of $E / N_{g}$ was estimated to be of the order of $10^{2} \mathrm{Td}$. The emission of discharge is mainly in violet and ultra violet range. The emission of $\mathrm{N}_{2}$ and $\mathrm{O}_{2}$ molecules and $\mathrm{OH}$ radical were observed in the near electrode regions and in the column. The emission of 
ion $\mathrm{N}_{2}^{+}$and hydrogen atom was registered in the near-cathode region only. In the infrared range the emission of oxygen atom was registered too.

At the present time our main interest is to examine the ultraviolet and the deep ultraviolet range of the discharge spectrum. So, the part of the experimental set-up for spectroscopic investigations was changed. We replaced the quartz lens by the spherical mirror to avoid the chromatic aberration. A block diagram of spectroscopic measurements is shown in figure 2.

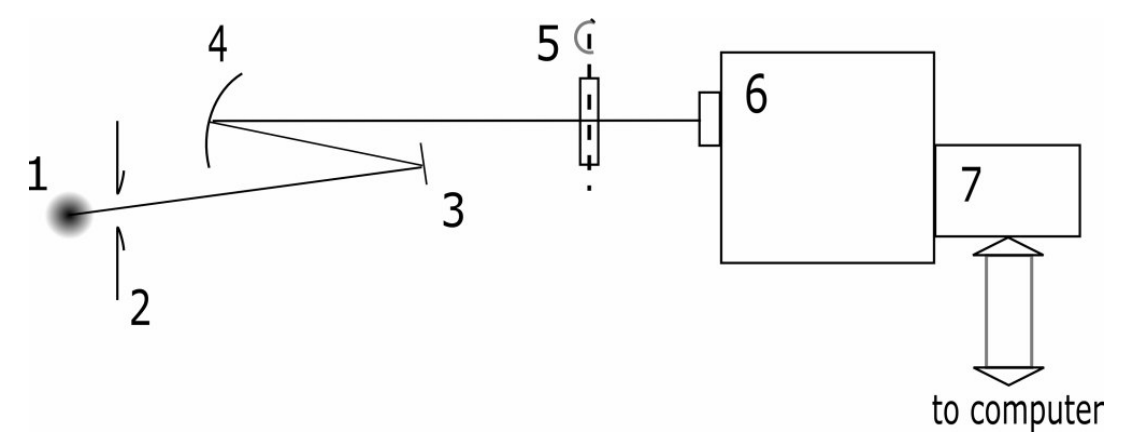

Figure 2: Block diagram of spectroscopic measurements. 1 discharge channel cross section; 2 diaphragm; 3 plain mirror; 4 spherical mirror; 5 plain-parallel quartz plate; 6 monochromator; 7 multichannel optical analyser with linear photodetector.

The necessity to use the plane mirror (3) is the result of some specific geometrical problems of our set-up. The spherical mirror (4) produced a reduced image of the discharge on the entrance slit of the monochromator (6), which is equipped with the grating of 1200 lines/mm providing the inverse dispersion of $1.3 \mathrm{~nm} / \mathrm{mm}$. The scanning of the image across the slit was carried out using a plain parallel quartz plate (5). A multichannel optical analyzer with a linear detector was used to record the radiation. The detector could record in a single measurement a part of spectrum of $22 \mathrm{~nm}$ in length. The spectral resolution with an entrance slit $0.1 \mathrm{~mm}$ is about $0.2 \mathrm{~nm}$. Spectral sensitivity of our set-up was measured with the help of calibrated band lamp with a sapphire window. Equipment used provided the possibility to calibrate discharge spectra up to $\lambda \geq 240 \mathrm{~nm}$.

The discharge with a length $L \approx 6.5 \mathrm{~mm}$ was examined. Each measurement of the spectrum was repeated three times. The results were summarized in the computer. This set-up permitted us to record spectra in the deep ultra violet range. Nevertheless, in this study, our main interest is the investigation of the part of the spectrum in the range $365-385 \mathrm{~nm}$, which contains $\mathrm{N}_{2}$ and $\mathrm{O}_{2}$ emission. 


\section{Molecular spectrum calculations}

In the range $365-385 \mathrm{~nm}$, the spectrum is composed of two summed spectra. The first is due to the second positive system of $N_{2}\left(C^{3} \Pi_{u}-B^{3} \Pi_{g}\right)$ and the second is due to the Schumann-Runge system of $\mathrm{O}_{2}\left(\mathrm{~B}^{3} \Sigma^{-}{ }_{\mathrm{u}}-\mathrm{X}^{3} \Sigma_{\mathrm{g}}^{-}\right)$. To simulate the spectrum we have to take into account both systems.

The wave numbers of a transition from an upper quantum state $\left(n^{\prime}, v^{\prime}, J^{\prime}\right)$ to a lower quantum state $\left(n^{\prime \prime}, v^{\prime \prime}, J^{\prime \prime}\right)$ is obtained by calculation of the energy difference between the two states taking into account the allowed quantum rules $[10,11,12]$ :

$$
\sigma_{n^{\prime \prime}, v^{\prime \prime}, J^{\prime \prime}}^{n^{\prime}, J^{\prime}}=T_{n^{\prime}}+G_{n^{\prime}}\left(v^{\prime}\right)+F_{n^{\prime}, v^{\prime}}\left(J^{\prime}\right)-\left[T_{n^{\prime \prime}}+G_{n^{\prime \prime}}\left(v^{\prime \prime}\right)+F_{n^{\prime \prime}, v^{\prime \prime}}\left(J^{\prime \prime}\right)\right]
$$

where $n$ is the electronic quantum number, $v$ the vibrational quantum number, $J$ is the rotational quantum number. The wave number and energy terms are expressed in $\mathrm{cm}^{-1} . T$ is the electronic excitation energy of the quantum level $(n), G$ is the vibrational energy of the quantum level $(n, v)$ and $F$ is the rotational energy of the quantum level $(n, v, J)$.

At each wave number, the integrated volume emission (intensity) is calculated by the following expression :

$$
\begin{aligned}
I_{n^{\prime \prime}, v^{\prime,}, J^{\prime \prime}}^{n^{\prime} v^{\prime}, J^{\prime}}= & C t e \times\left(\sigma_{n^{\prime \prime}, v^{\prime \prime}, J^{\prime \prime}}^{n^{\prime}, v^{\prime}, J^{\prime}}\right)^{4} \times S_{v^{\prime} v^{\prime \prime}} \times S_{J^{\prime} J^{\prime \prime}} \times \frac{\phi \times N_{n^{\prime}}}{\left(2-\delta_{0, \Lambda^{\prime}+\Lambda^{\prime \prime}}\right) \times\left(2 S^{\prime}+1\right)} \\
& \times \frac{\exp \left(-\beta\left[\frac{G\left(v^{\prime}\right)}{T_{v i b}}+\frac{F_{v^{\prime}}\left(J^{\prime}\right)}{T_{r o t}}\right]\right)}{Q_{\text {int }}\left(T_{r o t}, T_{v i b}\right)}
\end{aligned}
$$

where $\beta=\frac{h c}{k}$ is equal to $1.4387685 \mathrm{~cm} . \mathrm{K} . h, c, k$ respectively represent the Planck's constant, the velocity of light and the Boltzmann's constant. Cte is a constant. Factor $S_{v^{\prime} v^{\prime \prime}}$ is the strength band of the band $\left(v^{\prime}, v^{\prime \prime}\right)$. Our chosen values are given in table 1 . The strength line $S_{J^{\prime} J^{\prime \prime}}$ is the Höln-London factor, which is calculated from Kovacs' tables [10]. The internal partition function $Q_{i n t}$ depending on the rotational and vibrational temperatures is written as follows $[13,14]$ :

$$
Q_{\text {int }}\left(T_{r o t}, T_{v i b}\right)=\sum_{v=0}^{v_{\max }} \exp \left(-\beta \frac{G(v)}{T_{v i b}}\right) \sum_{J=0}^{J_{\max }(v)} \frac{g j}{\varsigma} \times \exp \left(-\beta \frac{F_{v}(J)}{T_{r o t}}\right)
$$

$N_{n^{\prime}}$ represents the density of the excited level $n^{\prime}$ of the diatomic molecule. $g_{J}$ is the rotational statistical weight and $\zeta$ is equal to 2 for $\mathrm{O}_{2}$ and $\mathrm{N}_{2}$. 
We have to note that equation (2) assumes two main assumptions. The first is that the population densities of vibrational and rotational levels satisfy the Boltzmann distribution at respectively $T_{v i b}$ and $T_{\text {rot }}$. The second is the Born-Oppenheimer approximation.

The calculated intensities (2) are convoluted with a Gaussian profile determined by its Full Width at Half-Maximum $(F W H M)$ that we take equal to $0.19 \mathrm{~nm}$. The contribution of all lines emitted is summarised in the considered spectral range. To obtain the wavelength from the wave number we take into account the index refraction of air [15]. Since the considered wavelength is not deep in ultraviolet, we do not take absorption into account $[15,16]$. Then the simulated spectrum of each considered system is obtained for the following parameters: rotational temperature $T_{r o t}$, vibrational temperature $T_{v i b}$ and upper level density $N_{n^{\prime}}$ of electronic excitation quantum level $n^{\prime}$. That is to say $\mathrm{C}^{3} \Pi_{\mathrm{u}}$ for $\mathrm{N}_{2}$ and $\mathrm{B}^{3} \Sigma^{-}$, for $\mathrm{O}_{2}$.

\begin{tabular}{|l|l|}
\hline$\left(v^{\prime}-v^{\prime \prime}\right)$ & $S_{v^{\prime} v^{\prime \prime}}\left(D^{2}\right)$ \\
\hline$(0-16)$ & 0.383241 \\
\hline$(0-17)$ & 0.243796 \\
\hline$(1-17)$ & 0.367441 \\
\hline$(2-17)$ & 0.015282 \\
\hline$(2-18)$ & 0.031089 \\
\hline$\left(v^{\prime}-v^{\prime \prime}\right)$ & $S_{v^{\prime} v^{\prime \prime}}\left(D^{2}\right)$ \\
\hline$(3-18)$ & 0.215482 \\
\hline$(4-19)$ & 0.093186 \\
\hline$(5-19)$ & 0.133834 \\
\hline$(6-20)$ & 0.091272 \\
\hline$(7-20)$ & 0.104947 \\
\hline
\end{tabular}

Table 1 a: $\mathrm{S}_{\mathrm{v}^{\prime} \mathrm{v}}$ strength bands values of the Schumann-Runge system of $\mathrm{O}_{2}$ taken from RADEN data base [17] used for the calculated spectra in $365-385 \mathrm{~nm}$ wavelength range, $D$ is the Debye unit.

\begin{tabular}{|l|l|}
\hline$\left(v^{\prime}-v^{\prime \prime}\right)$ & $S_{v^{\prime} v^{\prime \prime}}\left(\boldsymbol{D}^{2}\right)$ \\
\hline$(0-2)$ & 3.739799 \\
\hline$(1-3)$ & 4.985702 \\
\hline$(2-4)$ & 3.945647 \\
\hline$(3-5)$ & 2.238900 \\
\hline
\end{tabular}

Table 1 b: $S_{v^{\prime} v^{\prime \prime}}$ strength bands values of the second positive system of $\mathrm{N}_{2}$, sequence -2 taken from RADEN data base [17] used for the calculated spectra in 365-385 nm wavelength range. 


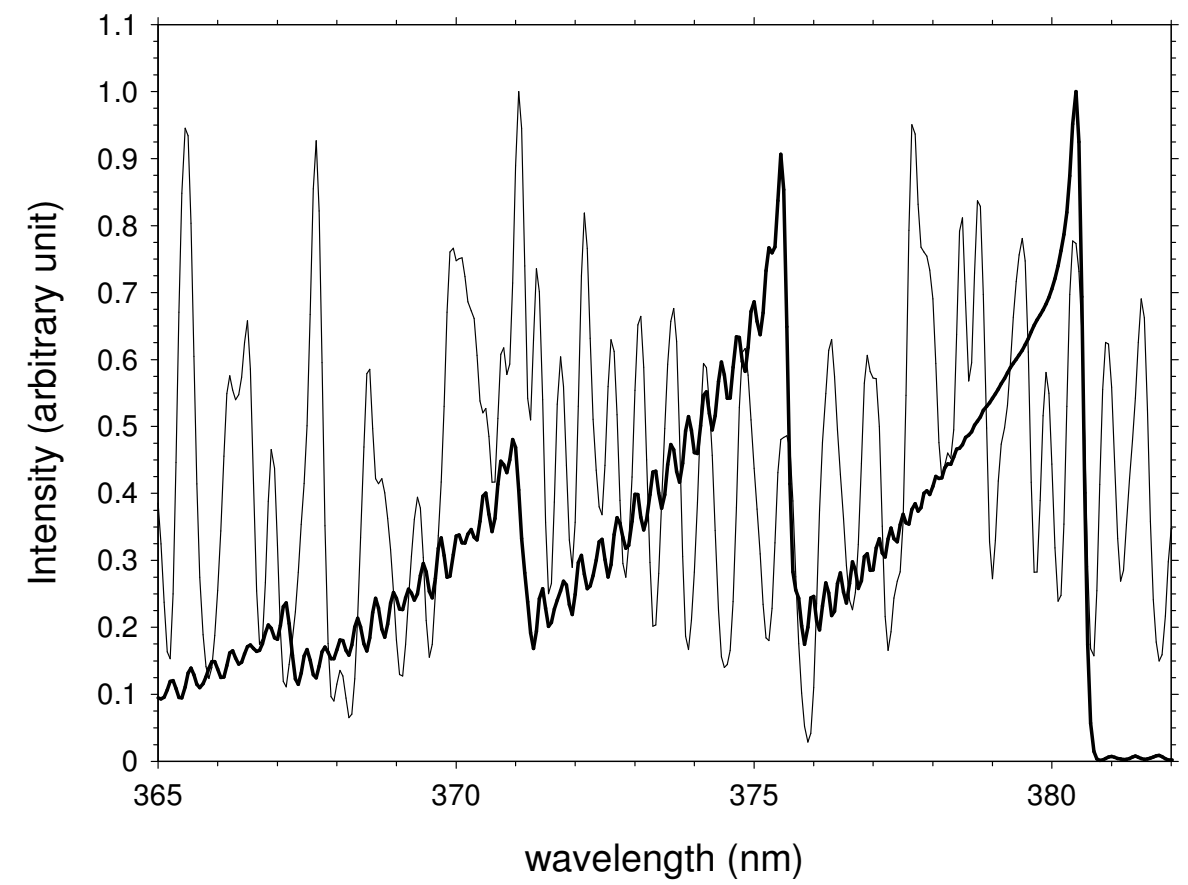

Figure 3 : Calculated intensities (arbitrary unit) of Schumann-Runge system of $\mathrm{O}_{2}\left(\mathrm{~B}^{3} \Sigma_{\mathrm{u}}^{-}\right.$$\mathrm{X}^{3} \Sigma_{\mathrm{g}}^{-}$) (thin line) and second positive system of $\mathrm{N}_{2}\left(\mathrm{C}^{3} \Pi_{\mathrm{u}}-\mathrm{B}^{3} \Pi_{\mathrm{g}}\right)$ (thick line) versus wavelength. Rotational temperature, vibrational temperature and FWHM are respectively fixed at $2000 \mathrm{~K}, 4000 \mathrm{~K}$ and $0.19 \mathrm{~nm}$.

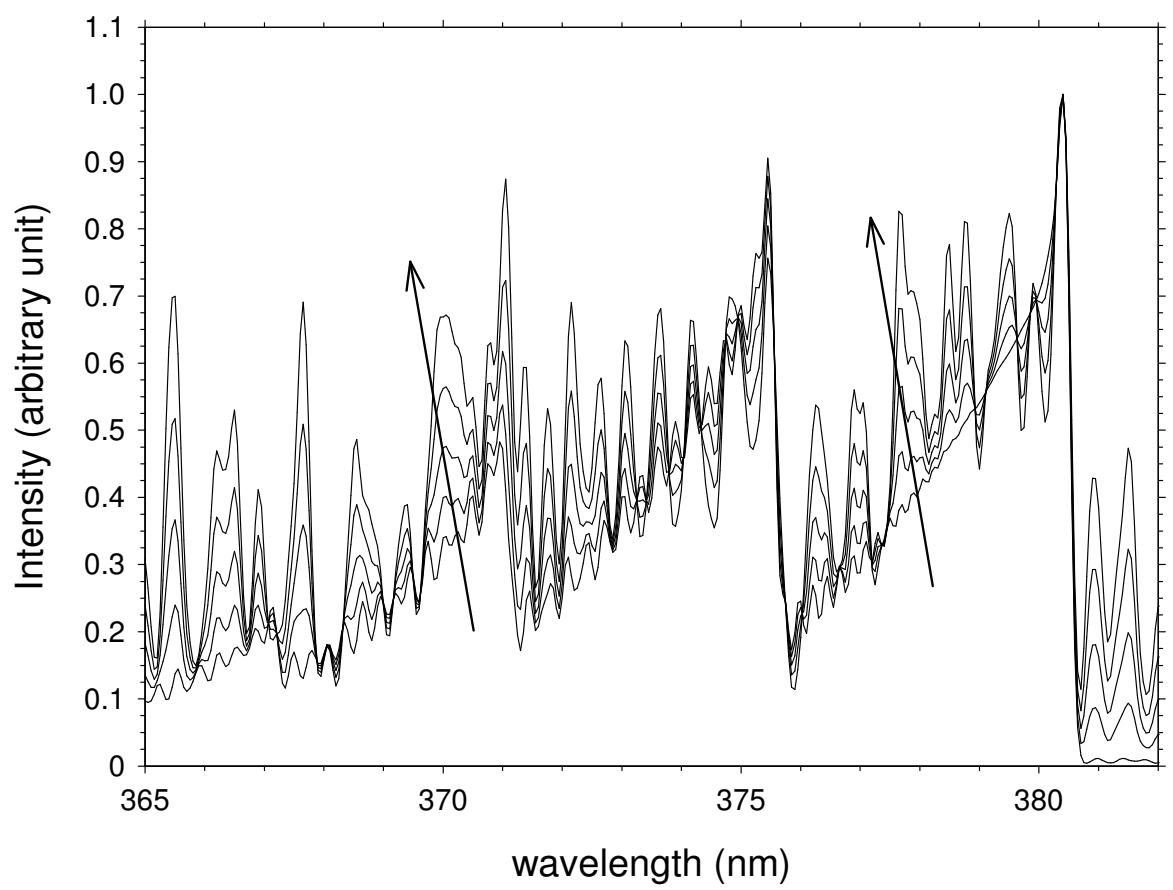

Figure 4 : Resulting Spectra (Schumann-Runge system of $\mathrm{O}_{2}$ and second positive system of $\left.\mathrm{N}_{2}\right)$ for several $\chi^{*}(0.01,0.20,0.50,1.00,2.00)$ values versus wavelength. $\left(T_{r o t}=2000 \mathrm{~K}\right.$, $\left.T_{v i b}=4000 \mathrm{~K}, \mathrm{FWHM}=0.19 \mathrm{~nm}\right)$. 
In figure 3 the spectra in arbitrary units of the intensity are plotted versus wavelength of both considered systems. The two spectra are presented separately to realize wavelength position of each system. We can notice that the systems perturb each other so the interpretation of experimental spectra is difficult. Consequently we introduce a new parameter $\chi^{*}$ that is the ratio between densities of two upper electronic quantum levels of the two considered systems:

$$
\chi^{*}=\frac{\left[O_{2}\left(B^{3} \Sigma_{u}^{-}\right)\right]}{\left[N_{2}\left(C^{3} \Pi_{u}\right)\right]}
$$

In figure 4, we have plotted the intensity of the resulting spectrum versus the wavelength for several ratios $\chi^{*}(0.01,0.2,0.5,1,2)$. The rotational temperature has been fixed to $2000 \mathrm{~K}$, and the vibrational temperature to $4000 \mathrm{~K}$. We note that the resulting spectrum depends mainly on the ratio $\chi^{*}$ and consequently on the density of the $C^{3} \Pi_{u}$ level for $N_{2}$ and $\mathrm{B}^{3} \Sigma_{\mathrm{u}}^{-}$level for $\mathrm{O}_{2}$. So, the comparison with experimental results is a new source of comprehensive results.

\section{Comparison between experimental and simulated spectra; results and discussion.}

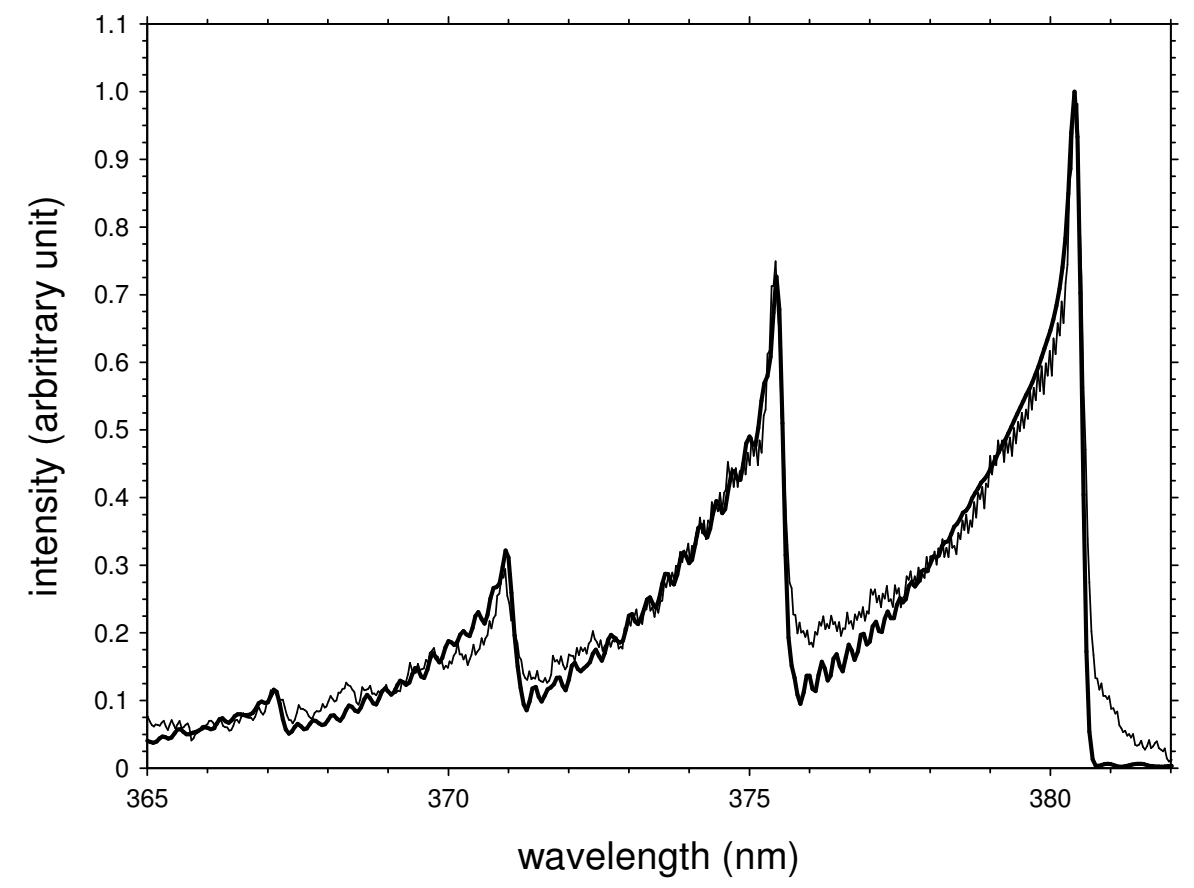

Figure 5 a: Comparison between the experimental spectrum (thin line) and the simulated spectrum (thick line) with the best fitting parameters $T_{r o t}, T_{v i b}$ and $\chi^{*}: 1400 \mathrm{~K}, 3400 \mathrm{~K}$ and 0.01 respectively at a distance of $1 \mathrm{~mm}$ from the anode. 


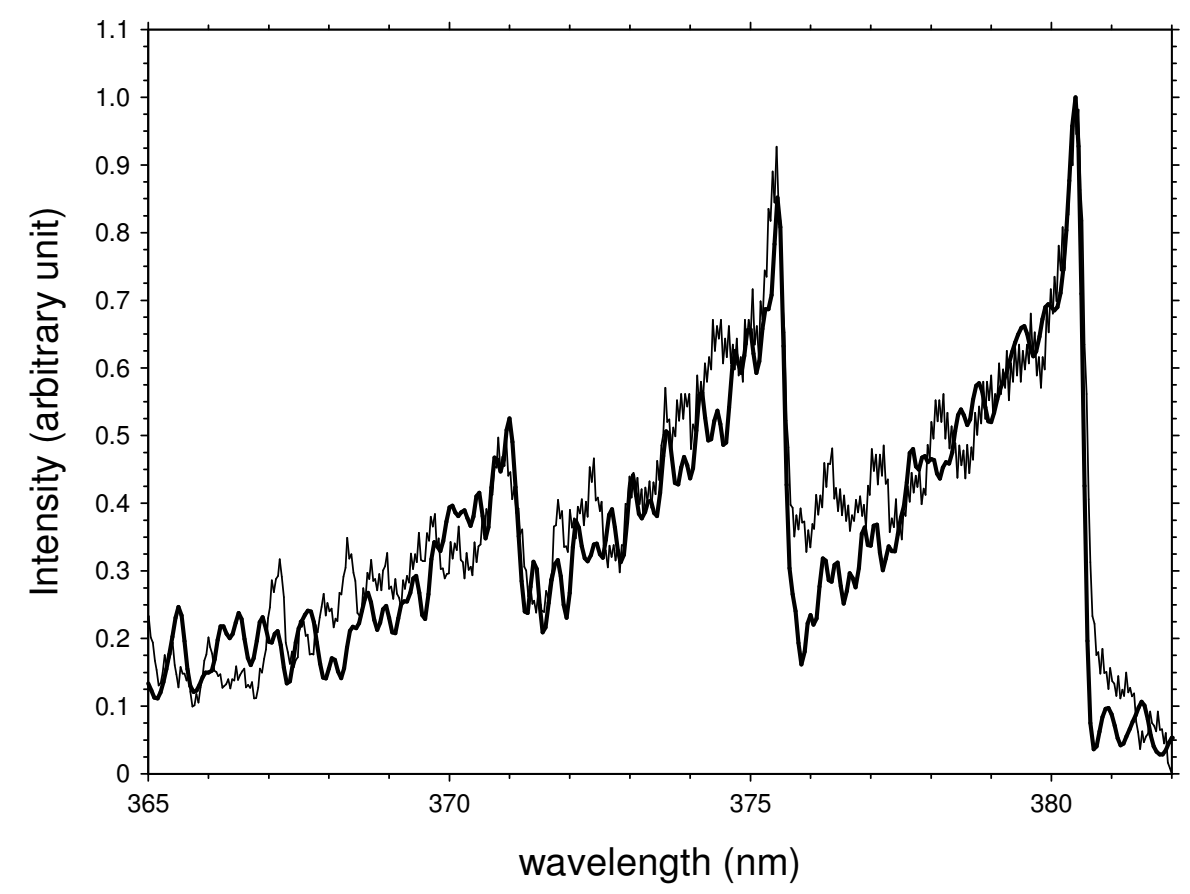

Figure 5 b: Comparison between the experimental spectrum (thin line) and the simulated spectrum (thick line) with the best fitting parameters $T_{r o t}, T_{v i b}$ and $\chi^{*}: 2000 \mathrm{~K}, 3800 \mathrm{~K}$ and 0.25 respectively at a distance of $1.9 \mathrm{~mm}$ from the anode.

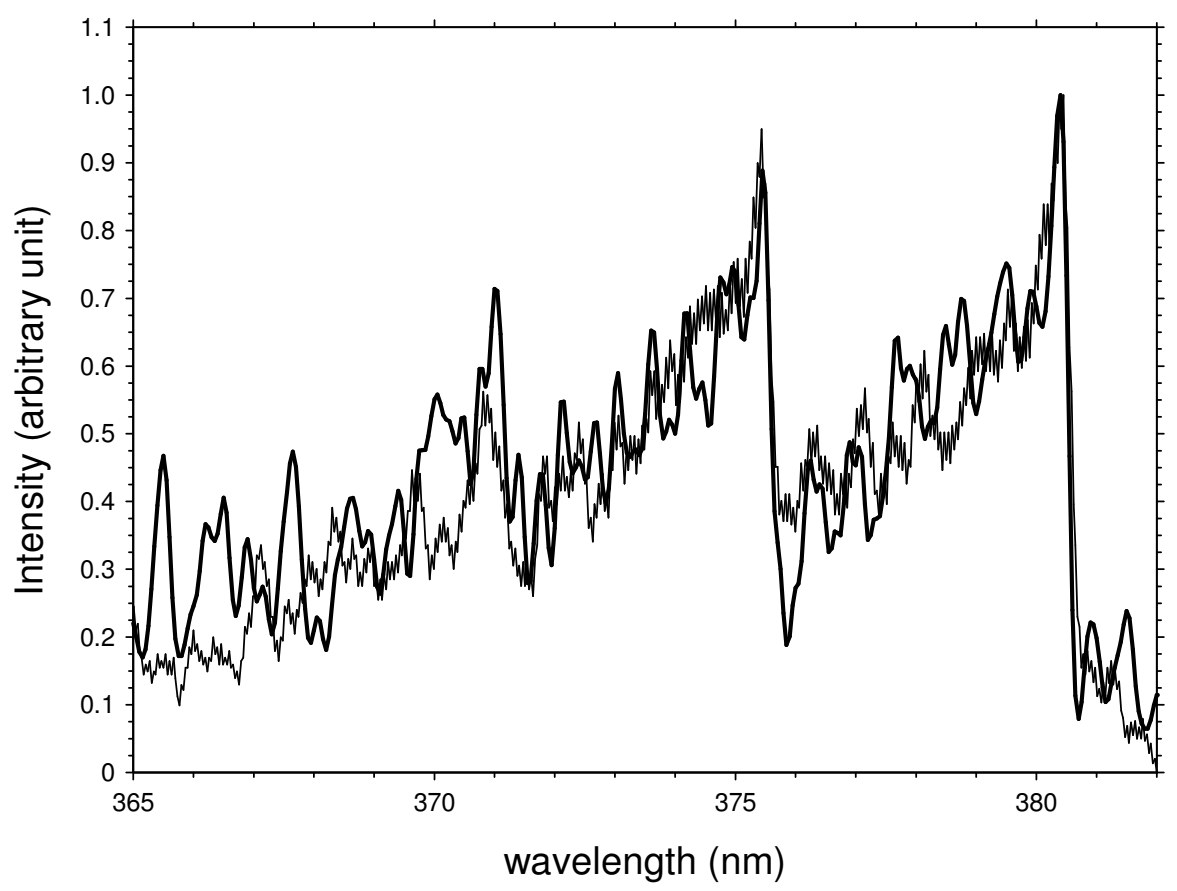

Figure 5 c: Comparison between the experimental spectrum (thin line) and the simulated spectrum (thick line) with the best fitting parameters $T_{r o t}, T_{v i b}$ and $\chi^{*}: 2400 \mathrm{~K}, 4000 \mathrm{~K}$ and 0.70 respectively at a distance of $4.2 \mathrm{~mm}$ from the anode. 


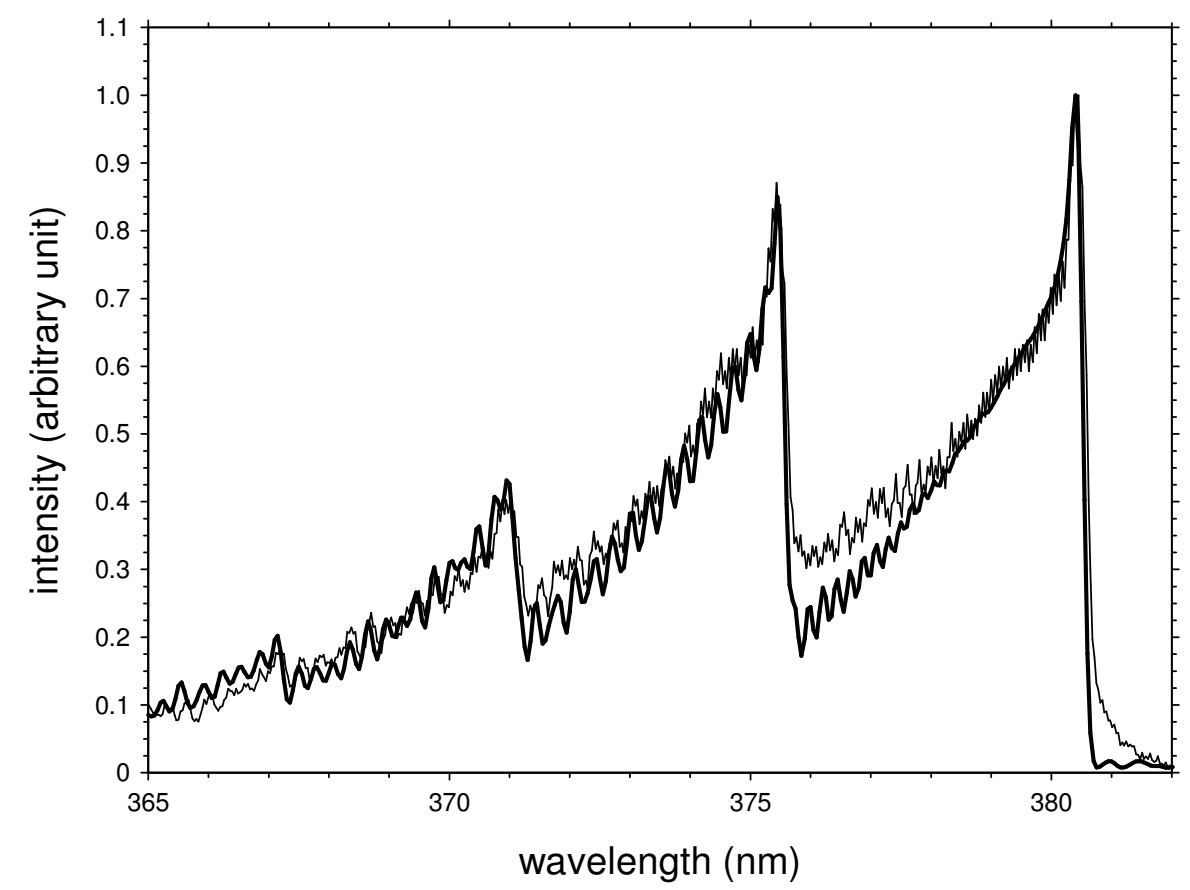

Figure 5 d: Comparison between the experimental spectrum (thin line) and the simulated spectrum (thick line) with the best fitting parameters $T_{r o t}, T_{v i b}$ and $\chi^{*}: 2000 \mathrm{~K}, 3600 \mathrm{~K}$ and 0.03 respectively at a distance of $5.6 \mathrm{~mm}$ from the anode.

The resulting simulated spectrum in the considered wavelength range depends on three parameters: the rotational temperature $T_{r o t}$, the vibrational temperature $T_{v i b}$ and the parameter $\chi^{*}$. By fitting the experimental results, we have tried to determine those parameters. For this purpose, we first determine $\chi^{*}$ from spectrum part in the wavelength range around $370 \mathrm{~nm}$ and around $377 \mathrm{~nm}$. Afterwards, with this value of $\chi^{*}$, we compare spectra for different values of $T_{r o t}$ in the $376-381 \mathrm{~nm}$ wavelength range. As a matter of fact the variation of $T_{v i b}$ has no effect on the spectra in this wavelength range if the spectra are normalized in intensity at the band-head (0-2) of $\mathrm{N}_{2}(2 \mathrm{~S}+)$. Finally, $T_{v i b}$ is determined in the wavelength interval of $365-$ $385 \mathrm{~nm}$. In figures 5 we show the result of the best fitting. We note that spectra shown in fig.5a and $5 \mathrm{~d}$ were measured in the near-electrode regions and spectra shown in fig. $5 \mathrm{~b}$ and $5 \mathrm{c}$ were measured in the discharge column.

It can be seen that the measured and simulated spectra are in rather good accordance. But there are some discrepancies in the details. 


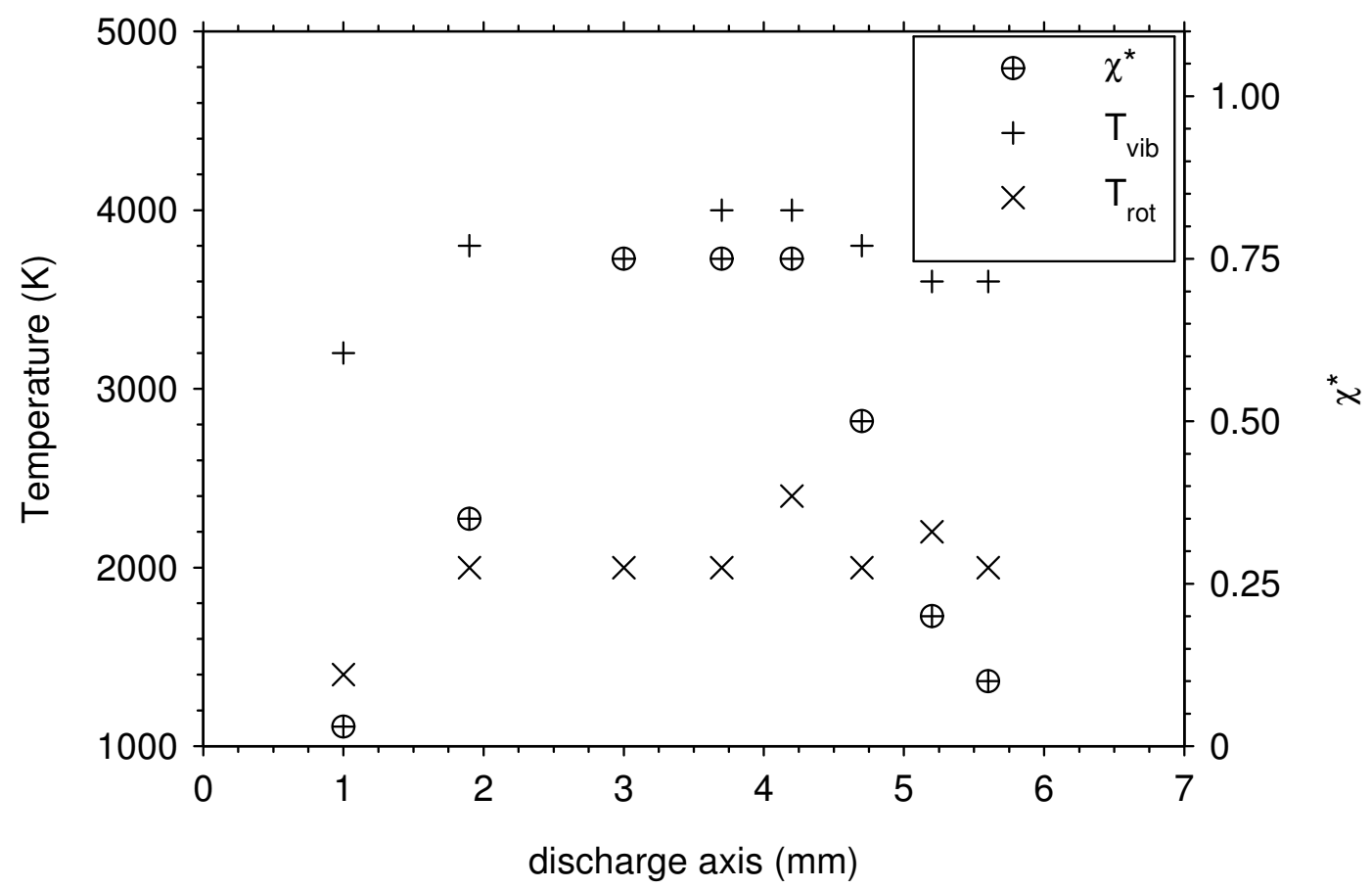

Figure 6: $\mathrm{T}_{\mathrm{vib}}, \mathrm{T}_{\text {rot }}$ and $\chi^{*}$ obtained along the discharge axis.

In figure 6 , we have plotted the axial distribution of obtained rotational and vibrational temperatures and $\chi^{*}$ parameters. It can be seen that the parameter $\chi^{*}$ is approximately constant in the main part of the column $(1.5 \leq \mathrm{z} \leq 4 \mathrm{~mm})$ and a small decrease can be observed in the near anode end. But in the near electrode regions the $\chi^{*}$ parameter decreases significantly. This behavior can be explained taking into account the axial dependences of the $\mathrm{N}_{2}$ and $\mathrm{O}_{2}$ emission intensities. $\mathrm{N}_{2}$ emission has pronounced near-electrodes maxima [7]. $\mathrm{O}_{2}$ emission is distributed much more homogeneously in the whole interelectrode gap and does not display maxima near the electrodes. As it was shown in our previous work [7], the electric field increases significantly in near-electrodes regions. So, one can conclude that the populations of $\mathrm{N}_{2}\left(\mathrm{C}^{3} \Pi_{\mathrm{u}}\right)$ and of $\mathrm{O}_{2}\left(\mathrm{~B}^{3} \Sigma_{\mathrm{u}}^{-}\right)$levels are determined by various processes.

In [8], the mean electron energy value in the plasma column is obtained by the comparison of the electrical probe and microwave emission absorption measurements with the results of the composition calculation. We use this value of electron energy to calculate the absolute value of the emission of some species $\left(\mathrm{N}_{2}, \mathrm{~N}_{2}{ }^{+}, \mathrm{O} \mathrm{I}, \mathrm{H}_{\alpha}\right)$. We have assumed that these levels are populated in accordance with Boltzmann distribution. The comparison with the results of spectroscopic measurements was in surprisingly good agreement. Calculating $\mathrm{O}_{2}$ spectra in our considered wavelength range with the same electron energy and the same 
assumption about Boltzmann distribution (the same calculation code with $T_{\text {rot }}=2100 \mathrm{~K}$, $T_{v i b}=3800 \mathrm{~K}$ ), we observe a great difference with experimental result (figure 7). To obtain a closed normalized spectra to experimental spectra, we have to decreases the total concentration of $\mathrm{O}_{2}$ by a factor of $510^{3}$ that is not acceptable taking our previous results into account [8]. We can conclude that $\mathrm{O}_{2}\left(\mathrm{~B}^{3} \Pi_{\mathrm{u}}\right)$ is not populated in accordance with Boltzman distribution at the above mentioned temperature.

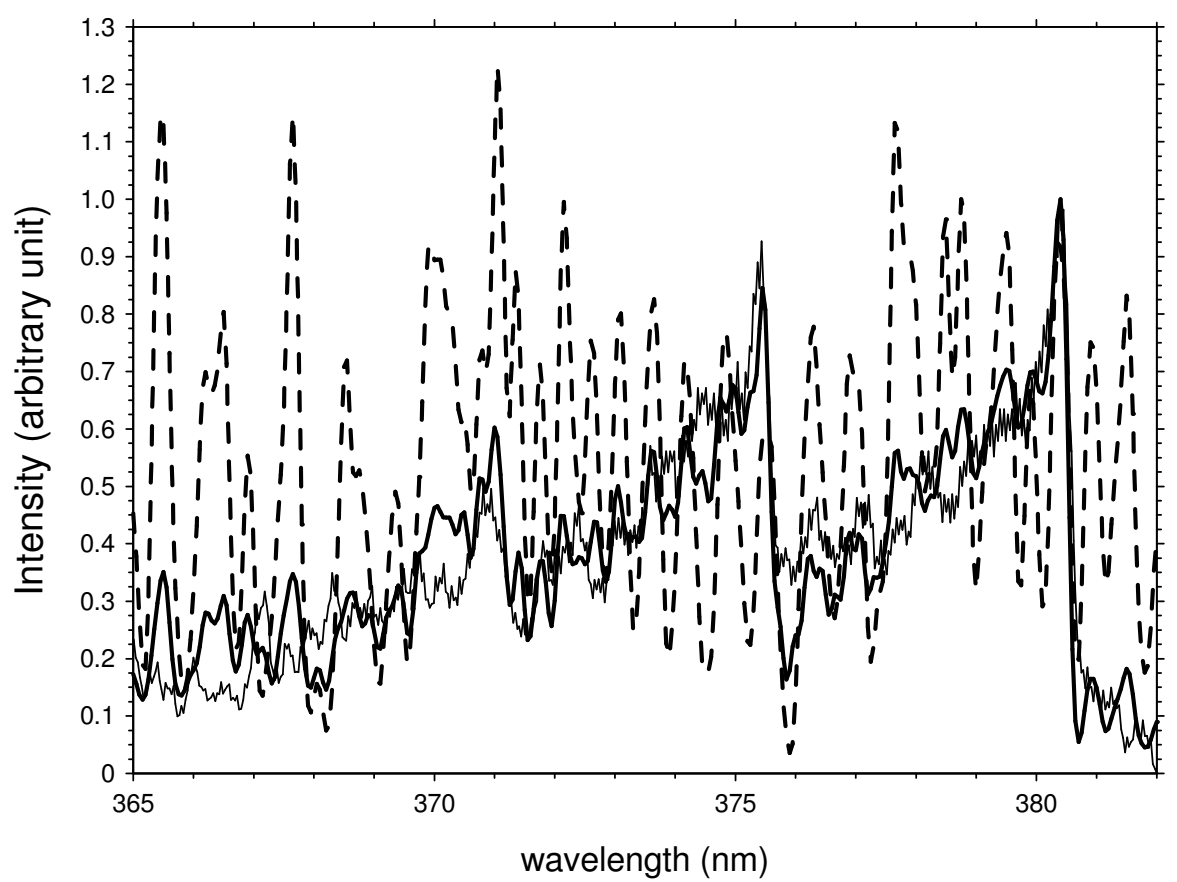

Figure 7: Comparison of normalized spectra. Experimental spectrum (distance of $1.9 \mathrm{~mm}$ from the anode) (Thin line), calculated spectra $\left(T_{r o t}=2100 \mathrm{~K}, T_{v i b}=3800 \mathrm{~K}, \mathrm{FWHM}=0.19 \mathrm{~nm}\right)$ with $\left[\mathrm{O}_{2}\right]=10^{24} \mathrm{~m}^{-3}$ and $\left[\mathrm{N}_{2}\right]=4 \quad 10^{24} \mathrm{~m}^{-3}$ (dashed curve) and with $\left[\mathrm{O}_{2}\right]=2 \quad 10^{20} \mathrm{~m}^{-3}$ and $\left[\mathrm{N}_{2}\right]=410^{24} \mathrm{~m}^{-3}$ (bold curve).

The rotational temperature and vibrational temperatures have a value respectively around $2100 \mathrm{~K}$ and around $3800 \mathrm{~K}$ in the main part of discharge column. The value of $T_{r o t}$ is in a good agreement with our previous results but the value of $T_{v i b}$ somewhat lower. $T_{v i b}$ was overestimated in [7] because in the past we did not take into account $\mathrm{O}_{2}$ emission. 


\section{Conclusion}

The results obtained in this work demonstrate that the comparison of spectra simulation with experimental spectra of DLMNE, which contain the second positive system of $\mathrm{N}_{2}$ and the Schumann-Runge system of $\mathrm{O}_{2}$ in the wavelength range of 365 to $385 \mathrm{~nm}$, give rather good results. But there exists some discrepancies in the details.

In our previous papers [7, 8], we showed a good agreement between the composition calculation, the measurements of the electrons concentration and the intensity of emission of some species $\left(\mathrm{N}_{2}(2+)\right.$ system, $\mathrm{N}_{2}{ }^{+}, \mathrm{O}$ I, $\left.\mathrm{H}_{\alpha}\right)$ in the column of DLMNE. We showed also that the water vapour proportion is high near the cathode zone and slowly decreases toward the anode.

Our new results show that the population of the $\mathrm{B}^{3} \Sigma^{-}$u of $\mathrm{O}_{2}$ does not follow the Boltzmann distribution assumed in the composition calculation. Some preliminary results of the measurements of $\mathrm{NO}(\mathrm{A})$ emission show analogous regularity. We'd like to note that the emissions of $\mathrm{N}_{2}, \mathrm{~N}_{2}{ }^{+}$, O I, $\mathrm{H}_{\alpha}$ mentioned above come from the levels with high excitation energy. On the contrary, $\mathrm{O}_{2}(\mathrm{~B}-\mathrm{X})$ and $\mathrm{NO}(\mathrm{A}-\mathrm{X})$ emissions come from the levels with lower electronic excitation energy. We intend to show the results of $\mathrm{NO}(\mathrm{A})$ emission investigations in our next publication.

So we can think that $\mathrm{O}_{2}(\mathrm{~B})$ and $\mathrm{NO}(\mathrm{A})$ are quenched with a species as $\mathrm{H}_{2} \mathrm{O}$ and/or $\mathrm{OH}$ because in our discharge exists a relatively high contamination by the water vapour. Furthermore, $\mathrm{H}_{2} \mathrm{O}$ and $\mathrm{OH}$ are known as effective quenchers $[18,19]$. We intend to prolong this work and to try to confirm the latter explanation proposed here.

\section{References}

[1] Chul Park, Overview of radiation problems in planetary entries, Proc. of the Int. Workshop on Radiation of High Temperature Gases in Atmospheric Entry, Porquerolles, France, 30 Sept. 2004, p3-14, ESA SP-583.

[2] Lino da Silva, M. Dudeck and R. Reis, Aerothermodynamics and ground test facilities simulation of planetary entry flows-Application to earth, Mars and Titan atmospheric entries, Proc. of the Int. Workshop on Planetary Probe Atmospheric Entry and Descent Trajectory Analysis and Science, Lisbonne, Portugal, 6 Oct. 2003, p 85-92, ESA SP-544.

[3] U. Kogelschatz, Industrial innovation based on fundamental physics, Plasma Sources Sci. Technol, Vol 11, A1-A6, 2002. 
[4] B. Benstaali, P. Boubert, B.G. Cheron, A. Addou and J.L. Brisset, Density and Rotational Temperature Mesurements of the OH and NO Radicals Produced by a Gliding Arc in Humid Air, PCPP, Vol 22, N4, 2002.

[5] C. Laux, T.G. Spence, C.H. Kruger, R.N. Zare, Optical diagnostics of atmospheric pressure air plasmas, Plasma Sources Sci. Technol, Vol 12, p 125-138, 2003

[6] B. Izrar, M. Dudeck, P. Andre, M.F. Elchinger, J. Aubreton, Supersonic Argon Flow In An Arc Plasma Source, AIP Conference Proceedings, 812, (1), p 355-358, 2006

[7] P. Andre, Yu. Barinov, G. Faure, V. Kaplan, A. Lefort, S. Shkol'nik, D. Vacher, Experimental study of discharge with liquid non-metallic (tap-water) electrodes in air at atmospheric pressure, J. Phys. D: Appl. Phys, Vol..34, p.3456-3465, 2001.

[8] P. Andre, J. Aubreton, Yu. Barinov, M.F. Elchinger, P.Fauchais, G. Faure, V. Kaplan, A.Lefort, S.Shkol'nik, Theoretical study of column of discharge with liquid non-metallic (tap water) electrodes in air at atmospheric pressure, J. Phys. D: Appl. Phys., Vol.35, p1846-1854, 2002.

[9] G. Faure, S.Shkol'nik, Determination of rotational and vibrational temperatures in a discharge with liquid non-metallic electrodes in air at atmospheric pressure, J. Phys. D, Vol 31, p 1212-1218, 1998.

[10] I. Kovacs, Rotational structure in the spectra of diatomic molecules, (1969) published by Adam Hilger LTD, London

[11] G. Herzberg, Molecular Spectra and Molecular Structure, I Spectra of Diatomic Molecules, D Van Nostrand Company, New York, 1950.

[12] K.P. Huber, G. Herzberg, "Molecular Spectra and Molecular Structure IV. Constants of Diatomic Molecules” Van Nostrand Reinhold Co., 1979.

[13] P. Andre, Partition functions and concentrations in plasmas out of thermal equilibrium IEEE Transactions on Plasma Science 23, issue 3, p 453-458, 1995.

[14] K.S. Drellishak, D.P. Aeschliman, A. B. Cambel, Partition Functions and Thermodynamic Properties of Nitrogen and Oxygen Plasmas, The Physics of Fluids, Vol 8, Nº, p 1590-1599, 1965.

[15] CRC Handbook of Chemistry and Physics 82nd ed (David R. Lide, ed.), CRC Press, New York, 2001.

[16] C. Laux, Optical Diagnostics and Radiative Emission of Air Plasmas, PhD, Stanford University, 1993.

[17] RADEN: The databank on radiative and energy parameters for diatomic molecules, Moscow State University, Chemical department. Ludmila Kuznetsova, http://www.elch.chem.msu.ru/raden/index.htm

[18] G. A. Raiche and D.R. Crosley, Temperature dependent quenching of the $A^{2} \Sigma^{+}$and $B^{2} \Pi$ states of NO, J Chem Phys, Vol 92,N¹, p 5211-5217, 1990.

[19] G. D. Greenblatt and A.R. Ravishankara, Collisional quenching of NO(A) by various gases, Chem. Phys. Letters, Vol 136, Nº, p 501-505, 1987. 